\title{
PROSPECTS FOR NEW PHYSICS AT THE LHC
}

\author{
John ELLIS* \\ Theory Division, Physics Department, CERN, \\ CH-1211 Geneva 23, Switzerland \\ *E-mail: John.Ellis@cern.ch
}

CERN-PH-TH/2010-074

\begin{abstract}
High-energy collisions at the LHC are now starting. The new physics agenda of the LHC is reviewed, with emphasis on the hunt for the Higgs boson (or whatever replaces it) and supersymmetry. In particular, the prospects for discovering new physics in the 2010-2011 run are discussed.
\end{abstract}

Keywords: LHC; Higgs boson; supersymmetry; extra dimensions.

\section{Preamble}

Back in the dawn of prehistory, when I was a student, Murray Gell-Mann was an inspiration to me. His work dominated the particle physics landscape that I entered then: strangeness, $V-A$ theory, the eightfold way, quarks, current algebra, the renormalization group, and so much more, and laid the basis for the developments that have occurred since. These fundamental contributions are now so embedded in the fabric of particle physics that perhaps we sometimes forget to remember them and celebrate their originator with all the respect he deserves. It is therefore a pleasure for me to participate in this meeting honouring Murray and his achievements, and a privilege to be given the opportunity to speak here about the next chapter in particle physics that is now unfolding.

\section{Open Questions Beyond the Standard Model}

There is a standard list of open questions beyond the Standard Model of particle physics. What is the origin of particle masses, and are they due to a single elementary Higgs boson, or to something else? Why are there so many types of matter particles, and is the answer to this question related to the origin of matter in the Universe? What is the nature of the cold dark matter that makes up some $80 \%$ of the matter in the Universe? How to unify the fundamental forces? How to construct a quantum theory of gravity?

The LHC may be able to address all these questions. One of its main motivations has been to solve the mass problem, and its experiments should tell us definitively 
whether or not there exists a Higgs boson resembling that in the Standard Model. There is a large class of models in which cold dark matter is composed of particles that were in thermal equilibrium in the early Universe, in which case they should weigh $\sim 1 \mathrm{TeV}$, and be produced at the LHC. One example of such a theory is supersymmetry, which would also assist in the unification of the fundamental forces. Measuring the masses of supersymmetric particles, if they exist, would be a great way of testing predictions based on such theories. Supersymmetry and extra dimensions are key aspects of string theory, the only promising candidate for a consistent quantum theory of gravity, which could be tested in very novel ways if the LHC produces microscopic black holes. What are the prospects that the LHC might cast light on these enticing scenarios?

\section{Hunt for the Higgs}

In the Standard Model, particles acquire their masses from couplings to a universal scalar field whose associated quantum, called the Higgs boson, has become the 'Holy Grail' of particle physics. Direct searches for the Higgs boson by experiments at the LEP accelerator established the lower bound $m_{h}>114.4 \mathrm{GeV} .{ }^{1}$ Precision electroweak data are sensitive to $m_{h}$ through quantum corrections, and yield the preferred range $^{2}$

$$
m_{h}=87_{-26}^{+35} \mathrm{GeV} \text {. }
$$

The $95 \%$ confidence-level upper limit on the mass of the Higgs boson is $157 \mathrm{GeV}$ if only the precision electroweak data are used, or $186 \mathrm{GeV}$ if the LEP direct lower limit is included. Recently, the Tevatron experiments CDF and D0 have excluded a range of heavier masses: ${ }^{3}$

$$
162 \mathrm{GeV}<m_{h}<166 \mathrm{GeV} .
$$

A combined fit to all the data, shown in Fig. 1, yields the asymmetric estimate ${ }^{4}$

$$
m_{h}=116.4_{-1.3}^{+15.6} \mathrm{GeV}
$$

at the $68 \%$ confidence level.

If the Higgs mass is large, so is the Higgs quartic self-coupling $\lambda$, and renormalization-group effects cause it to blow up at some relatively low scale $\Lambda$, as seen in Fig. 2, heralding the appearance of new non-perturbative physics. On the other hand, if $m_{h}$ is small, negative renormalization by the large $t$-quark Yukawa coupling drives $\lambda<0$, leading to an instability in the electroweak vacuum, unless new physics such as supersymmetry intervenes. ${ }^{5}$ Only a narrow range of $m_{h} \in(130,180) \mathrm{GeV}$ is compatible with the survival of the Standard Model at all scales up to the Planck mass. This would be the 'maximal conceivable disaster' scenario for the LHC: a single Standard Model Higgs boson and nothing else! The precision electroweak data favour small values of $m_{h}$, and the combination with the Tevatron exclusion (2) excludes the blow-up scenario at the $99 \%$ confidence level. $^{6}$ The unstable-vacuum scenario is preferred, but the 'disaster' scenario is not even disfavoured at the 1- $\sigma$ level. 


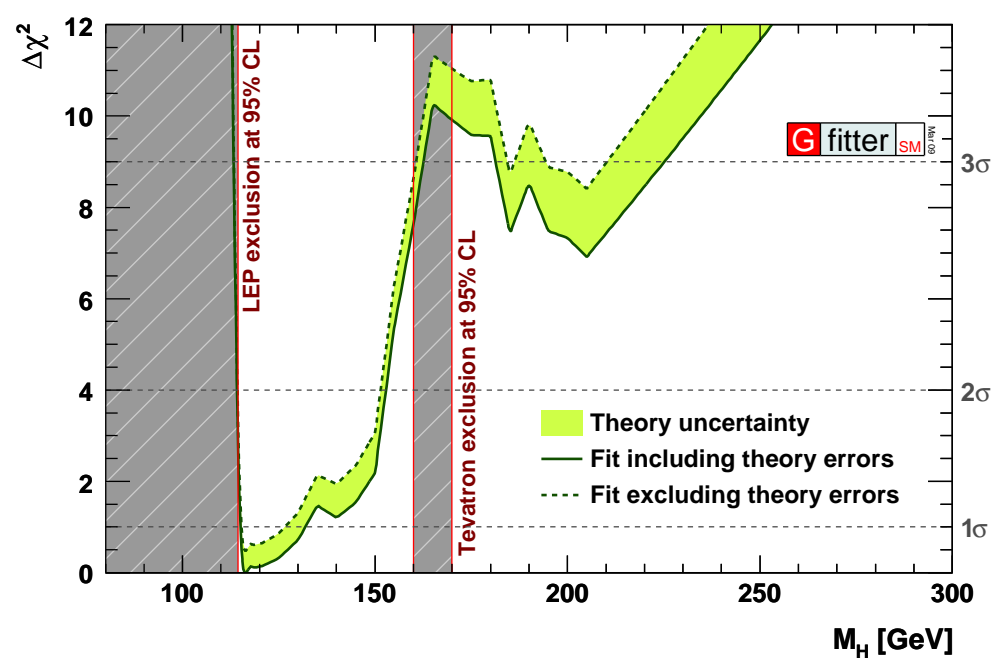

Fig. 1. The $\chi^{2}$ function for the Standard Model as a function of the Higgs mass, combining ${ }^{4}$ the precision electroweak data ${ }^{2}$ with the $\mathrm{LEP}^{1}$ and Tevatron ${ }^{3}$ exclusions.

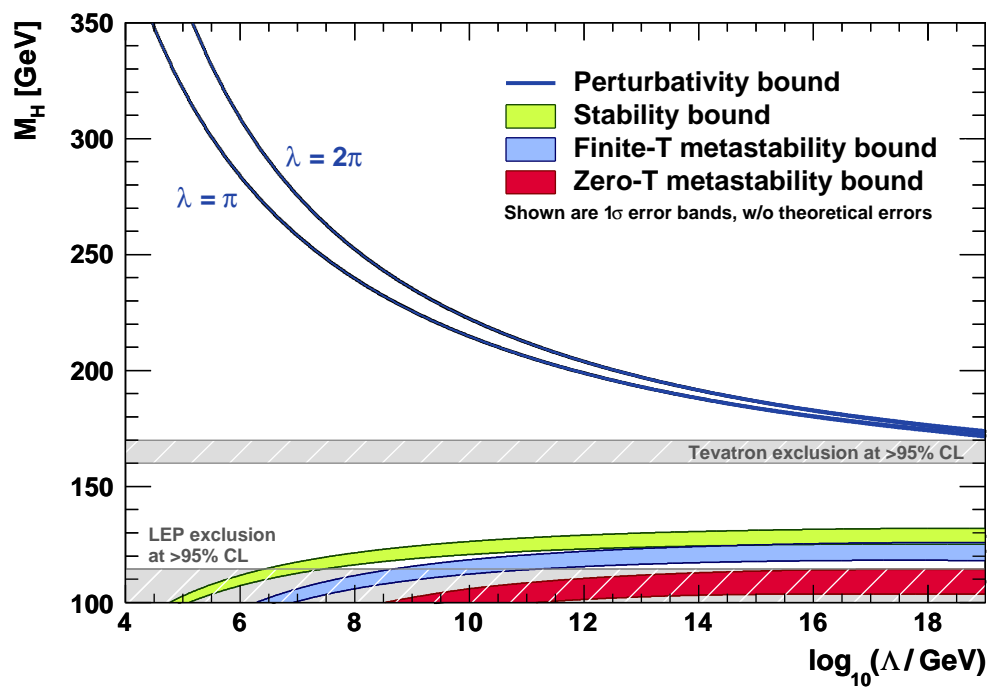

Fig. 2. If the Standard Model Higgs boson weighs more than $\sim 180 \mathrm{GeV}$, the Higgs self-coupling blows up at some scale $\Lambda$ below the Planck scale, inducing new non-perturbative physics. If it weighs less than $\sim 130 \mathrm{GeV}$, our current electroweak vacuum is unstable. The data summarized in Fig. 1 disfavour the blow-up scenario at the $99 \%$ confidence level. ${ }^{6}$

\section{The LHC Physics Haystack}

The standard list of the primary physics objectives of the LHC includes the search for this Higgs boson (or whatever replaces it), the nature of dark matter, the pri- 
mordial plasma that filled the Universe when it was less than a microsecond old, and matter-antimatter asymmetry. It is worth remembering that the cross sections for producing interesting heavy new particles at the LHC are typically $\mathcal{O}(1 / \mathrm{TeV})^{2}$ and possibly with additional factors $\sim \alpha^{2}$, much less than the total cross section $\mathcal{O}\left(1 / m_{\pi}\right)^{2}$. Typical cross sections for producing the Higgs boson or supersymmetric particles are $\sim 10^{-12}$ of the total cross section: looking for them will resemble searching for a needle in 100,000 haystacks!

\section{The Search for the Higgs Boson}

For this reason, the Higgs boson will not appear as soon as the LHC produces high-energy collisions. The left panel of Fig. 3 shows the amount of luminosity required at $14 \mathrm{TeV}$ in the centre of mass either to exclude the Higgs boson at the $95 \%$ confidence level or to claim a $5-\sigma$ discovery, as a function of its mass. We see that a couple of hundred inverse picobarns could get the LHC into the exclusion business, while several inverse femtobarns would be needed to guarantee detection at any mass. There is an intermediate range of masses $m_{h} \in(150,500) \mathrm{GeV}$ where detection via $h \rightarrow W W, Z Z$ decays is relatively easy, but in the preferred low-mass range (3) these decay modes are less important and Higgs detection becomes more difficult.
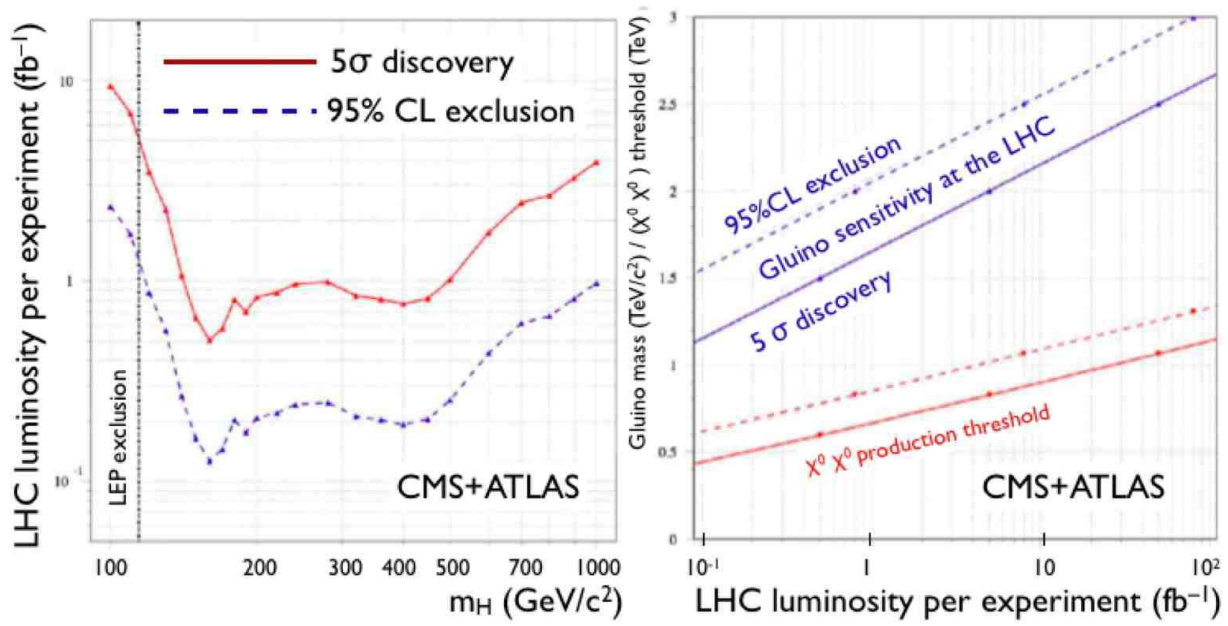

Fig. 3. Left panel: The sensitivity of the LHC running at $14 \mathrm{TeV}$ for $95 \%$ confidence level exclusion of the Standard Model Higgs boson (lower, dash-dotted curve) and 5- $\sigma$ discovery (upper, solid curve). Right panel: In the constrained MSSM, the mass of the gluino (which may be excluded or discovered at the LHC, upper lines) is correlated with the threshold for producing supersymmetric particles at a linear $e^{+} e^{-}$collider (lower lines). ${ }^{7}$

The stakes in the Higgs search are high, since it is key to many puzzles in cosmology as well as particle physics. How is electroweak symmetry broken? Is there 
such a thing as an elementary scalar field? What is the fate of the Standard Model at high scales? Did mass appear when the Universe was a picosecond old through an electroweak phase transition? Did CP-violating Higgs interactions help create the matter in the Universe? Did another elementary scalar field, the inflaton, cause (near-)exponential expansion of the early Universe, and hence make the Universe so big and old? The typical scale of vacuum (dark) energy in the Higgs potential is some 60 orders of magnitude larger than the measured value: why is there so little dark energy? Discovering the Higgs boson, or proving that it does not exist, may not answer all these questions, but it may be our best experimental probe of them.

The most exciting Higgs scenario for the LHC may be that it is proven not to exist. That would really shake us smug theorists out of our torpor! The best alternative known to me would be to break electroweak symmetry by boundary conditions in extra dimensions. ${ }^{8}$ Finding evidence for extra dimensions might be easier to explain to a lay audience than an ill-named God particle.

\section{The Search for Supersymmetry}

There are many motivations for supersymmetry: its beauty, it would render the hierarchy of mass scales more natural, it predicts a light Higgs boson, it stabilizes the electroweak vacuum, it facilitates grand unification, and it is apparently needed for the consistency of string theory. Here I focus on the fact that supersymmetry could provide the dark matter required by astrophysics and cosmology. ${ }^{9}$

In many supersymmetric models, there is a multiplicatively-conserved quantum number called $R$-parity, that may be represented as $R=(-1)^{2 S-L+3 B}$, where $S$ is spin, $L$ is lepton number, and $B$ is baryon number. It is easy to verify that known particles have $R=+1$, whereas their putative supersymmetric partners, differing in spin by $1 / 2$, would have $R=-1$. The conservation of $R$ parity would imply that sparticles are produced in pairs, that heavier sparticles decay into lighter ones, and that the lightest supersymmetric particle (LSP) is stable, because it has no legal decay mode. Hence, it should still be around after being produced early in the Big Bang, and could provide the needed dark matter. Presumably, the LSP is some neutral, weakly-interacting particle, otherwise it would have bound to ordinary matter and been detected by now. In this case, the favoured signature for supersymmetry at colliders is missing transverse energy carried away by the invisible dark matter particles.

There are important constraints on supersymmetry due to the absence of sparticles at LEP and the Tevatron, the LEP lower limit on $m_{h}$ and the consistency of $b$-quark decays with the Standard Model. Some hint of new physics at the TeV scale may be provided by the measurement ${ }^{10}$ of the anomalous magnetic moment of the muon, $g_{\mu}-2$, that could be explained by supersymmetry, although there are still uncertainties in the Standard Model calculation of $g_{\mu}-2 .{ }^{11}$ The measured density of dark matter, $0.097<\Omega_{D M} h^{2}<0.122$, provides a very tight constraint on some combination of supersymmetric model parameters, if the LSP provides the dark 
matter. The interplay of these constraints is shown in Fig. 4 for one particularly simple supersymmetric model with universal supersymmetry-breaking parameters $m_{1 / 2}$ and $m_{0}$ assumed at the GUT scale, the CMSSM. ${ }^{12}$

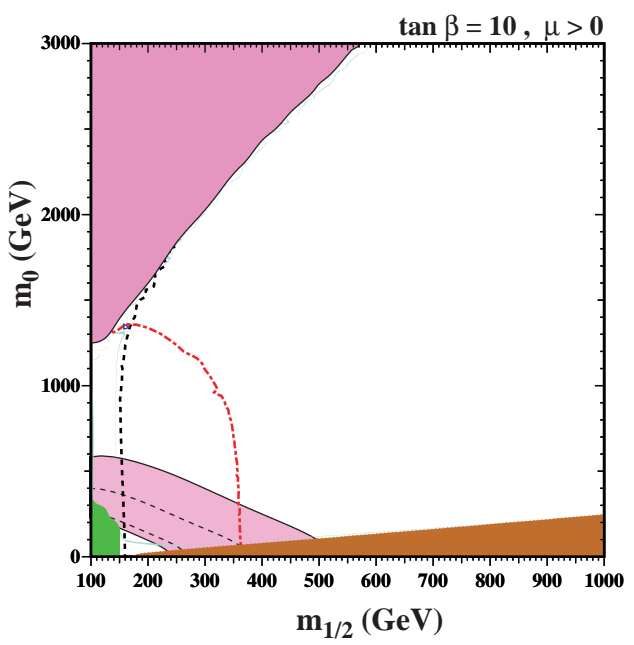

Fig. 4. The $\left(m_{1 / 2}, m_{0}\right)$ plane of the CMSSM for sample values of the other supersymmetric model parameters, showing the different theoretical, phenomenological, experimental and cosmological constraints. There is no consistent electroweak vacuum in the dark pink shaded region at large $m_{0}$, the LSP would be charged in the brown shaded region at small $m_{0}, b \rightarrow s \gamma$ excludes the green shaded region, LEP excludes the regions to the left of the dashed black and red lines by unsuccessful chargino and Higgs searches, respectively, and $g_{\mu}-2$ favours the paler shaded pink region. The LSP would have the appropriate cosmological density in the narrow turquoise strip close to the boundaries of the allowed region. ${ }^{12}$

\section{Where is Supersymmetry?}

We have recently made a global supersymmetric fit using a frequentist approach to analyze the precision electroweak data, the LEP Higgs mass limit, the cold dark matter density, $b$-decay data and (optionally) $g_{\mu}-2$. We combined the likelihood functions from these different observables to construct a global likelihood that can be used to infer preferred regions of the supersymmetric parameter space. ${ }^{13,14}$

We see in Fig. 5 that the preferred regions of the $\left(m_{0}, m_{1 / 2}\right)$ planes in both the CMSSM and a model with common non-universal supersymmetry-breaking contributions to the Higgs masses (NUHM1) correspond to relatively low masses where the relic LSP density is brought into the WMAP range by coannihilations with light sleptons, particularly the lighter stau. The 'focus-point' region at large $m_{0}$ is disfavoured, principally but not exclusively by $g_{\mu}-2$. If one drops this constraint, considerably larger ranges of $m_{0}$ and $m_{1 / 2}$ would be allowed, though small values are slightly preferred by other data. ${ }^{13,14}$ 
Fig. 6 compares the preferred regions of these $\left(m_{0}, m_{1 / 2}\right)$ planes with the $5-\sigma$ discovery reach of the LHC with given amounts of integrated luminosity at certain centre-of-mass energies. The $5-\sigma$ discovery reach of the LHC with $1 / \mathrm{fb}$ of luminosity at $7 \mathrm{TeV}$ in the centre of mass would probably include all the $68 \%$ confidence-level regions in Fig. 6.

If supersymmetric particles are this light, they would contribute significantly to the electroweak radiative corrections that test the Standard Model at the quantum
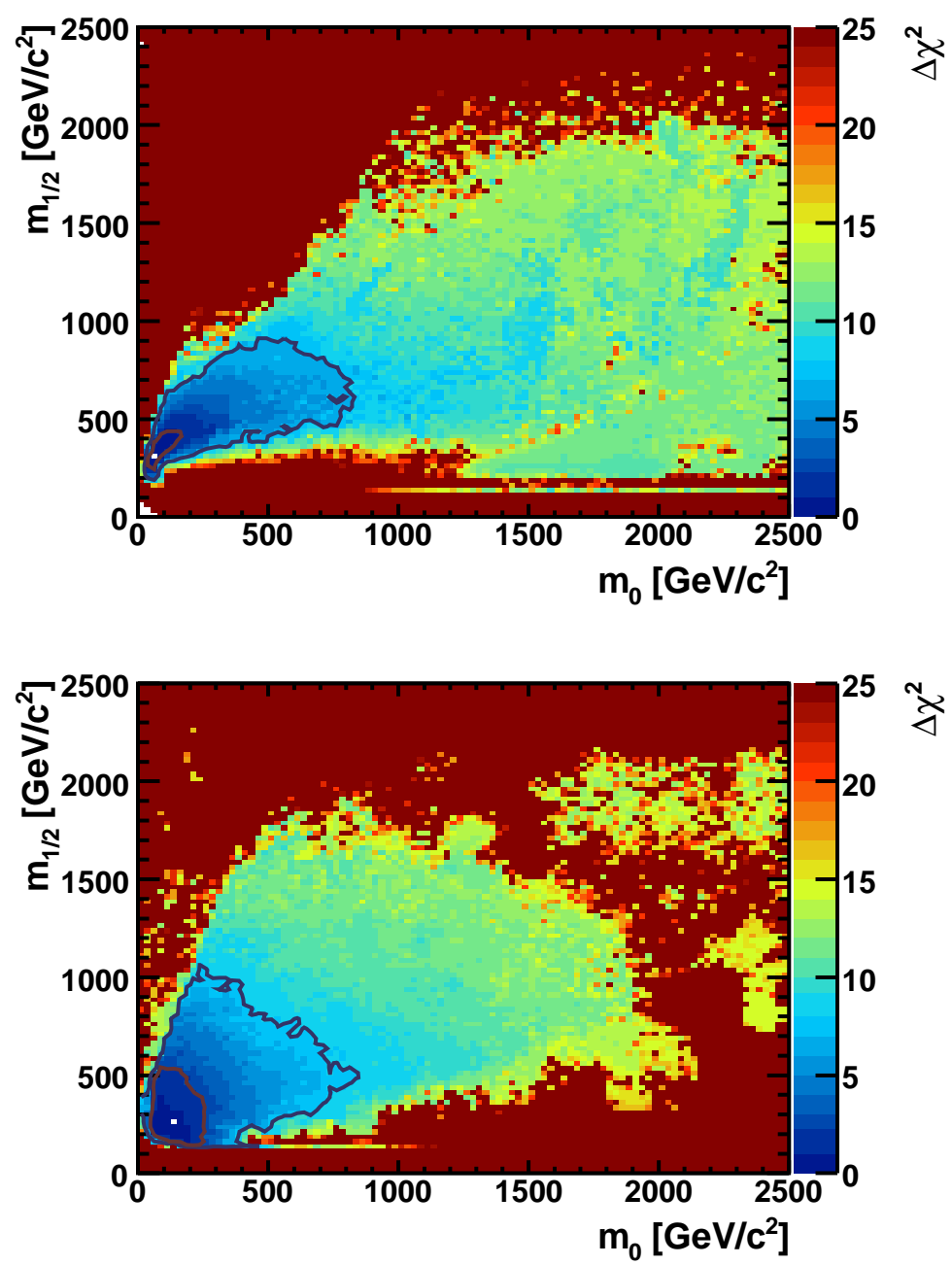

Fig. 5. The preferred regions in the $\left(m_{0}, m_{1 / 2}\right)$ planes of the CMSSM (upper panel) and the NUHM1 (lower panel), as found in a frequentist analysis. ${ }^{14}$ The best-fit points are shown as white points, and the $68 \%$ and $95 \%$ confidence-level contours are shown as solid black lines. 
level. Using low-energy precision measurements as input, one may use radiative corrections to predict high-energy observables such as $m_{t}, M_{W}$ and $m_{h}$. Within the Standard Model, the predictions of $m_{t}$ and $M_{W}$ have been very successful, and one would hope that their success would not be undermined in supersymmetric extensions of the Standard Model. On the one hand, supersymmetry predicts a restricted range for $m_{h}$, tending to reduce an uncertainty in the calculations of the radiative corrections. On the other hand, light sparticles could themselves contribute significantly to the radiative corrections.

As seen in Fig. 7, the net effect of supersymmetry in the context of the global fits to the CMSSM and NUHM1 introduced above is to reduce the predicted ranges of $m_{t}$ and $M_{W} \cdot{ }^{15}$ The predictions are in both cases in good agreement with the
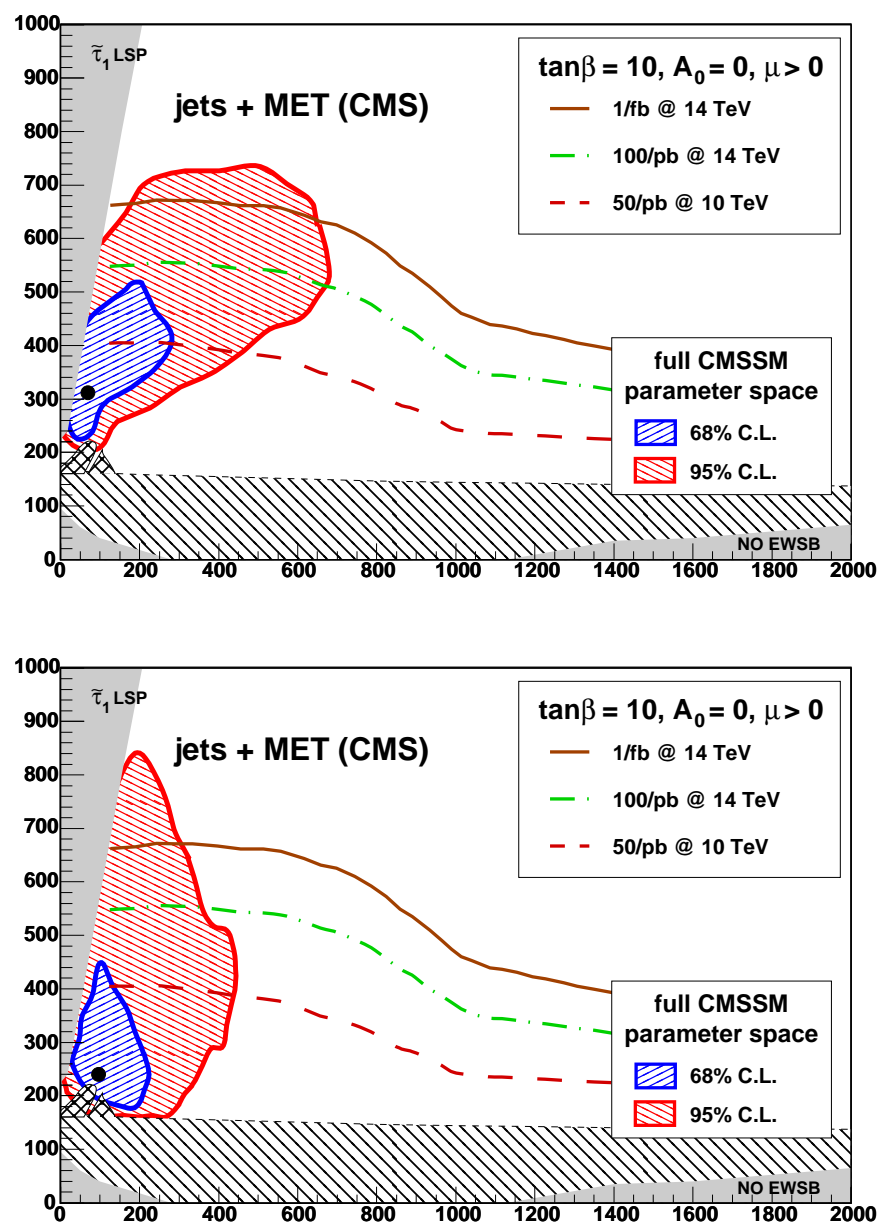

Fig. 6. The preferred regions in the $\left(m_{0}, m_{1 / 2}\right)$ planes of the CMSSM (upper panel) and the NUHM1 (lower panel), compared with the estimated discovery sensitivity of the LHC with different amounts of luminosity and centre-of-mass energy. ${ }^{13}$ 
measured values: indeed, the supersymmetric predictions for $M_{W}$ are even slightly better than those in the Standard Model.

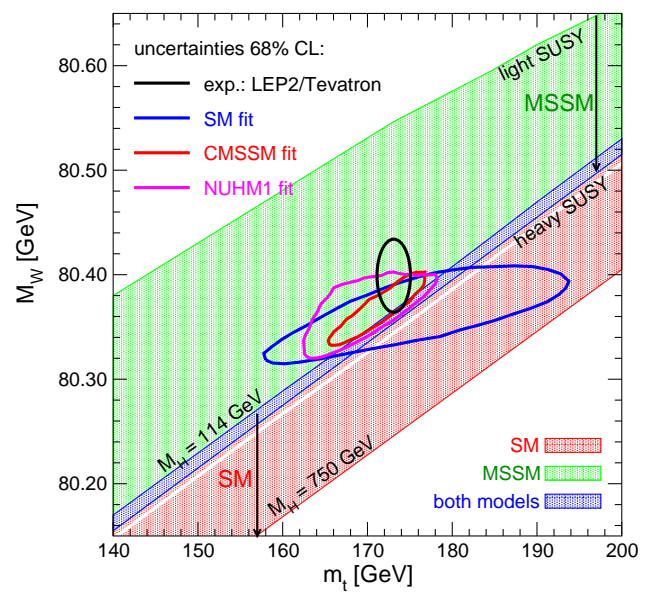

Fig. 7. Predictions for $m_{t}$ and $M_{W}$ on the basis of low-energy precision data in the Standard Model (blue curve), the CMSSM (red curve) and the NUHM1 (violet curve) compared with the experimental measurements at LEP and the Tevatron (black ellipse). ${ }^{15}$

\section{Extra Dimensions?}

Another fashionable scenario for physics beyond the Standard Model is the appearance of large extra dimensions. Their possible existence was suggested decades ago, and given extra momentum by string theory, which appears to require their appearance at some distance scale. However, our current understanding of string theory does not offer any firm guidance as to their possible sizes. It has been suggested that, if they are large they might solve or at least alleviate the mass hierarchy problem. Perhaps I am showing my age, but I find large extra dimensions less attractive than supersymmetry. Nevertheless, the final arbiters will be experiments, particularly at the LHC.

They may find Kaluza-Klein excitations of Standard Model particles, or they may discover missing energy leaking into extra dimensions, or they may discover that gravity becomes strong at the TeV scale and LHC collisions produce microscopic black holes. This last speculation has sparked concerns that have no scientific basis. The same theories that predict microscopic black holes also predict that they decay via Hawking radiation, with interesting grey-body factors that would be fascinating tests of string theory or any other quantum theory of gravity. Even if they were stable, the continued existence of the Earth and other celestial objects tells us to fear nothing from the LHC. ${ }^{16}$ Do I hear the sound of other axes being ground? 


\section{The Restart of the LHC}

Following the first start of the LHC on September 10th, 2008 and the electrical fault nine days later that laid the LHC low, there was jubilation on November 20th, 2009 when the LHC was restarted. This was redoubled 3 days later when the first collisions were observed at $900 \mathrm{GeV}$ in the centre of mass. Soon afterwards, the beams were successfully ramped up to $1.18 \mathrm{TeV}$ and the highest-energy humanmade collisions were seen.

Remarkably quickly, the LHC experiments were able to reconstruct the decays of known particles and remeasure their masses. Two-photon decays of the $\pi^{0}$ and $\eta$ were quickly seen, but seeing the Higgs will take a while, as discussed above: no Higgs yet! Both ATLAS and CMS have seen multi-jet events, and have shown distributions of the missing transverse energy. So far, these agree only too well with Monte Carlo simulations: no supersymmetry yet! Multi-jet events occur at rates compatible with QCD: no Hawking-decaying black holes yet!

\section{What will the Future Bring?}

While I was writing up this talk, the LHC produced its first collisions at $3.5 \mathrm{TeV}$ per beam on March 30th, 2010, and Fig. 8 shows some of the first events observed by the ATLAS and CMS experiments. The default LHC operating scenario is to collide at $3.5 \mathrm{TeV}$ per beam until the end of 2011 , aiming to accumulate $1 / \mathrm{fb}$ of integrated luminosity. Depending on the LHC running experience during this period, the LHC beam energy may be increased slightly during this period. As illustrated in Fig. 9, this should enable the LHC to at least equal the Tevatron sensitivity to an intermediate-mass Higgs boson weighing between 150 and $180 \mathrm{GeV}$. Moreover, it would give the LHC a reach for new physics such as supersymmetry that would extend beyond the Tevatron. Indeed, by discovering or excluding the gluino, this first physics run of the LHC may already be able to tell us whether supersymmetric are light enough to be produced with a $500 \mathrm{GeV}$ linear $e^{+} e^{-}$collider. $^{7}$

A long shutdown is then planned for consolidation of the LHC and its injectors, including the LHC magnet interconnects and training of the dipole magnets, making it possible to run the LHC at or close to its design energy of $7 \mathrm{TeV}$ per beam. Further in the future, there will be at least major upgrade of the LHC, incorporating Linac4 and new interaction-region insertions. The scope of possible further upgrades (an SPL? a higher extraction energy for the PS booster? replacement of the PS? new collision insertions? crab cavities?) are still under discussion, and will be decided only in the light of operational experience with the LHC.

\section{A Conversation with Mrs Thatcher}

Back in 1982, while she was Prime Minister of the UK, Mrs. Thatcher visited CERN and was introduced to British physicists. When she was told I was a theoretical physicist, she asked me "What do you do?" I explained that my job was to think 

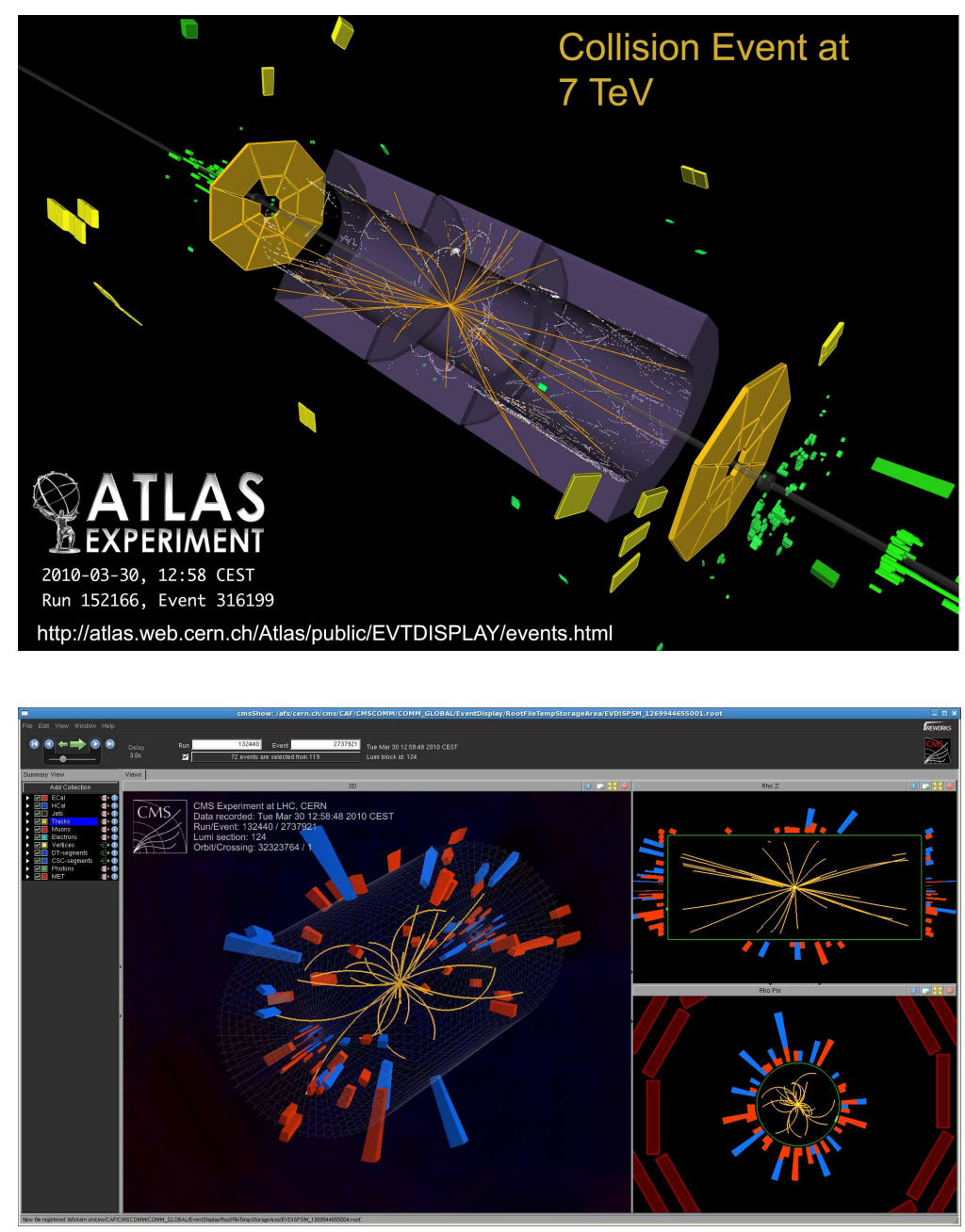

Fig. 8. First 7-TeV collisions from ATLAS (upper panel) ${ }^{17}$ and CMS (lower panel). ${ }^{18}$

of things for the experiments to look for, and hope they find something different. "Wouldn't it be better if they found what you predicted?" asked Mrs. T., who always liked her ideas to be vindicated. My response was that, in that case, we would not be learning anything really new. Likewise, I sincerely hope that the LHC will be remembered in the history of physics for something not described in this talk.

\section{Acknowledgments}

It is a pleasure to thank Harald Fritzsch for his kind invitation to this enjoyable event, and K. K. Phua and his team for their generous hospitality. Above all, it is a pleasure to thank Murray for his inspiration over the years. 

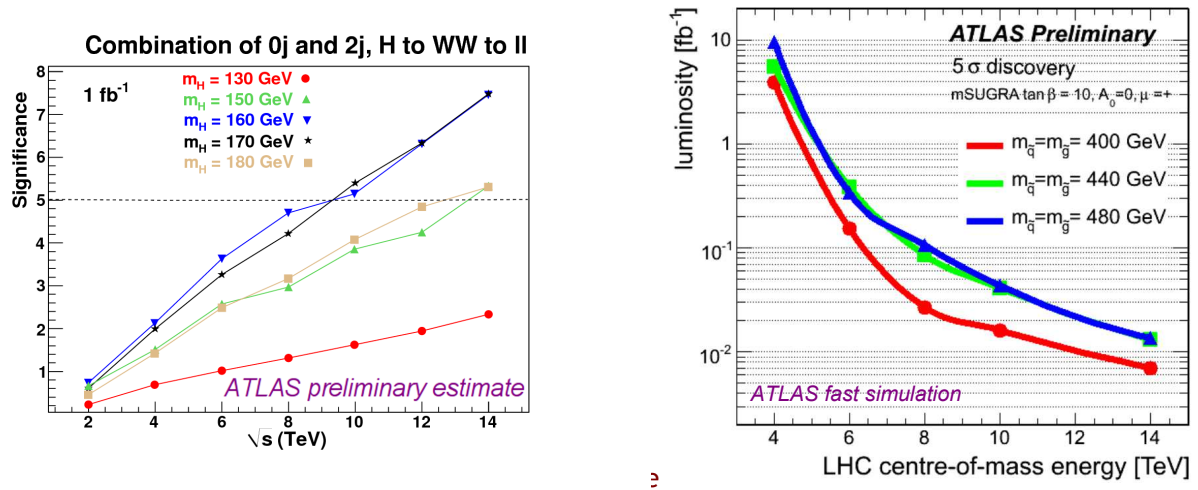

Fig. 9. Left panel: The ATLAS signal sensitivity for a Standard Model Higgs boson with 1/fb integrated luminosity at various centre-of-mass energies. Right panel: The sensitivity of ATLAS for 5 - $\sigma$ discovery of supersymmetric particles at reduced centre-of-mass energies. ${ }^{19}$

\section{References}

1. R. Barate et al. [ALEPH, DELPHI, L3 and OPAL Collaborations, LEP Working Group for Higgs boson searches], Phys. Lett. B 565 (2003) 61 [arXiv:hep-ex/0306033].

2. ALEPH, CDF, D0, DELPHI, L3, OPAL and SLD Collaborations, LEP Electroweak Working Group, Tevatron Electroweak Working Group and SLD electroweak and heavy flavour groups, arXiv:0911.2604 [hep-ex].

3. T. Aaltonen et al. [CDF and D0 Collaborations], Phys. Rev. Lett. 104 (2010) 061802 [arXiv:1001.4162 [hep-ex]].

4. H. Flacher, M. Goebel, J. Haller, A. Hocker, K. Moenig and J. Stelzer, Eur. Phys. J. C 60 (2009) 543 [arXiv:0811.0009 [hep-ph]].

5. J. R. Ellis and D. Ross, Phys. Lett. B 506 (2001) 331 [arXiv:hep-ph/0012067].

6. J. Ellis, J. R. Espinosa, G. F. Giudice, A. Hoecker and A. Riotto, Phys. Lett. B 679 (2009) 369 [arXiv:0906.0954 [hep-ph]].

7. J.-J. Blaising, A. De Roeck, J. Ellis, F. Gianotti, P. Janot, G. Rolandi and D. Schlatter, http://council-strategygroup.web.cern.ch/council-strategygroup/BB2/ contributions/Blaising2.pdf.

8. See, for example, C. Grojean, New theories for the Fermi scale, arXiv:0910.4976 [hep$\mathrm{ph}]$ and references therein.

9. J. R. Ellis, J. S. Hagelin, D. V. Nanopoulos, K. A. Olive and M. Srednicki, Nucl. Phys. B 238 (1984) 453.

10. G. W. Bennett et al., Phys. Rev. D73, 072003, 2006, hep-ex/0602035. 
11. M. Davier, A. Hoecker, B. Malaescu, C. Z. Yuan and Z. Zhang, arXiv:0908.4300 [hep$\mathrm{ph}]$.

12. J. Ellis and K. A. Olive, Chapter 8 of 'Particle Dark Matter: Observations, Models and Searches' edited by Gianfranco Bertone, pp. 142-163, Cambridge University Press Hardback ISBN 9780521763684, arXiv:1001.3651 [astro-ph.CO].

13. O. Buchmueller et al., JHEP 0809 (2008) 117 [arXiv:0808.4128 [hep-ph]].

14. O. Buchmueller et al., Eur. Phys. J. C 64 (2009) 391 [arXiv:0907.5568 [hep-ph]].

15. O. Buchmueller et al., Phys. Rev. D 81 (2010) 035009 [arXiv:0912.1036 [hep-ph]].

16. S. B. Giddings and M. L. Mangano, Phys. Rev. D 78 (2008) 035009 [arXiv:0806.3381 [hep-ph]]; J. R. Ellis, G. Giudice, M. L. Mangano, I. Tkachev and U. Wiedemann, J. Phys. G 35 (2008) 115004 [arXiv:0806.3414 [hep-ph]].

17. ATLAS Collaboration, http://atlas.web.cern.ch/Atlas/public/EVTDISPLAY/events.html.

18. CMS Collaboration, http://cms . web.cern.ch/cms/News/e-commentary/cms-e-commentary10.htm.

19. Updated from G. Aad et al. [ATLAS Collaboration], "Expected Performance of the ATLAS Experiment - Detector, Trigger and Physics," arXiv:0901.0512 [hep-ex]. 